Historic, Archive Document

Do not assume content reflects current scientific knowledge, policies, or practices. 



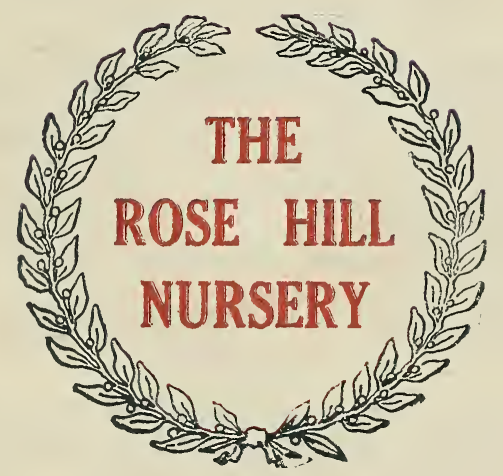

S. S. Shimer, Proprietor, Annamoriah, West Va. GRANTSVILLE NEWS PRINT 
$11^{\circ}$

1 i

$\because \div \quad \cdots$ 


\section{Rose Hill Nursery Price List}

These prices are for high grade nursery stock. All trees etc. guaranteed to be delivered in perfect growing condition. Satisfacticn guaranteed.

We will not be responsible for stock that becomes damaged after date of delivery. We do, however, agree to replace at one-half price, all stock that fails to live, providing purchaser receives it promptly, and plants and cares for it properly. Pürchaser must notify the Rose Hill Nursery Co. by August first after delivery, giving list of stock that failed to live and a duplicate of his order must accompany said notice.

We will pay freight charges on all orders amounting to ten dollars or more.

We can send grapes, berries, roses, shrubs, hulbs, tubers, and also one-year-old fruit and ornamental trees by Parcels Post at prices given in this list.

\section{Certificate of Inspection}

Our nurseries are inspecte 1 each year by inspectors of the Entomological Division of the Department of Agriculture of the Strte of West Virg nir, and are free from insect pests and diseases. A certificate of the department is attached to all shipments, and will also be furnished to $\mathrm{c}$ istomers if desired.

\section{How You Can Grow a Successful Orchard}

To grow a successful orchard a few impurtant points should be given careful consideration. To start right is most important, as a mistake at the beginning can never be correr.ted.

Plant Proper Varieties. - Select varieties that, are adapted to your section of the rountry. We make a specialty of growiny varieties suited to this sertion of country. If in duubt as to best varieties for vour lucation, write us, or your Experiment Station. This service is absilutely free.

Plant Gnod Varieties. - Buy sturdy, healthy, properly grown trees, with a good root system. Get trees grown in your neighborhood, if it is possible to get good trees close home. They will be acclimated then. That is a head and shoulder start in the race for profits. Read a good paper.

\section{APPLES}

Special Varieties.

Two or 1 hree years old 5 in $7 \mathrm{ft}$. 4 to $5 \mathrm{ft}$. 3 to $4 \mathrm{ft}$.

Each $\quad 10 \quad 100$ $70 \mathrm{c} \quad \$ 6.50 \quad \$ 60$

$\begin{array}{lll}60 \mathrm{c} & 5.50 & 50\end{array}$

$50 \mathrm{c} \quad 4.50 \quad 40$ 
One year old trees.

4 to $5 \mathrm{ft}$

$60 \mathrm{c}$

5.50

50

3 to $4 \mathrm{ft}$

$50 \mathrm{c}$

4.50

40

Albemarl Pippin, Banana (wirter), Delicious, Engiish Rambo, Ensee (Improved Rome Beauty), Golden Delightful, (iolden Winesap, Liveland Raspberry, Mother (Fall Delicious), Opalescent) Parks Seedless, Pound Royal, Rume Beauty, Stayman Winesap, Sweet Bough, Virginia Beauty.

Leading Varieties.

Two or three year old trees. Each $10 \quad 100$ 5 to $7 \mathrm{ft}$

4 to $5 \mathrm{ft}$ (i0c $\quad \$ 5.50 \quad \$ 50$

3 to $4 \mathrm{ft}$ 50 c $\quad 450 \quad 40$

One year old trees.

$4 \mathrm{t}, 5 \mathrm{ft}$ $40 \mathrm{c} \quad 3.60 \quad 30$

3 to $4 \mathrm{ft}$

$\begin{array}{lll}40 \mathrm{c} & 3.60 & 30\end{array}$ $30 \mathrm{c} \quad 2.70 \quad 20$

Aikin, Arkansas, Arkansas Black, Baldwin, Bailey Sweet, Ben Davis, Bismarck, Blankenship Sweet, Black Ben, Bónoni, Champion, Duchess of Oldenberg, Early Harvest, Early Russian, Early Stıawberry, Falliwater, Fall Pippin, Fall Rambo, Fall Wine, Gano, Golden Rusisett, Golden Sweet, June Sweet, King David, Lady Sweet, Maiden Blush, McIntosh Red, Mammoth Black Twig, Mo Pippin, Newtown Fippin, Northern spy, N. W. Greening, Oliver Red, Okabena, Pumpkin Sweet, Pryor Red, Red Astrachan, Red June, R. I. Greening, Stark, Senatnr, Summer Pearmain, Tallman Sweet, Wagner, Wealthy, Grimes Golden, Grindstone, Hedges Seedling. Ingram, leffreys, Jeniton, Jondthan, Winesap, Willow Twig, Wolf Kiver, Wonding's Favorite, Yellow Bell Flower, Yellow Transparent, York Imperial.

SPECIAL RATE:-When buying apple, pear, vench, quince, cherry, crabapple, plum, and dwarf vear, the following explanation should be read carefully:

100 Rate -50 or mure trees of a kind, as 50 appie, $50 \mathrm{p}+$ ar, etc.

10 Rare -10 or more of a variety at 10 rate. but if as many as 50 or more, customer is entitled to 100 rate.

Each Rate-Less tnan 10 of any variety at each rate.

\section{CRAB APPLE}

2 or 3 Year Old Trees. $\quad$ Each $10 \quad 100$

5 to $7 \mathrm{ft}$

4 to $5 \mathrm{ft}$

$3 \mathrm{ton} 4 \mathrm{ft}$

One Year Old Trees.

4 to $5 \mathrm{ft}$

3 to $4 \mathrm{ft}$

$60 \mathrm{c} \quad \$ 550 \quad \$ 50$

$\begin{array}{lll}50 \mathrm{c} & 4.50 & 40\end{array}$

$40 \mathrm{c} \quad 3.60 \quad 30$

Florence, Gen. Graut, Hyslop, Improved Native, Martha, Transcendant, Whitney. 
5 to $7 \mathrm{ft}$

4 to $5 \mathrm{ft}$

3 to $4 \mathrm{ft}$

One Year Old Trees.

5 to $6 \mathrm{ft}$

4 to $5 \mathrm{ft}$

3 to $4 \mathrm{ft}$ *Duchess Garber, *Howell, Jap. Golden Russett, Knonce Krull, *Keiffer. Lawrence, Le Coute, Lincoln, *Ros«ney, *Rutter Seedless, Seck+1 (Sugar), Snyder, Wilder Ey.

All Pears starred $\left(^{*}\right)$ can also be supplied as dwarfs at same price as standurds.

\section{QUINCE}

4 to $5 \mathrm{ft}$

3 to $4 . \mathrm{ft}$

2 to $3 \mathrm{ft}$
Each $\quad 10 \quad 100$

$\$ 1 . \quad \$ 9.00 \quad \$ 80$

$90 \mathrm{c} \quad 8.00 \quad 70$

80 c $\quad 7.00 \quad 60$

90 c $\quad 8.00 \quad 70$

$80 \mathrm{c} \quad 7.00 \quad 60$

$70 \mathrm{c} \quad 6.00 \quad 50$

Borgeat, Mechs Prolific, Mo. Mammoth, Orange.

\section{CHERRY}

$\begin{array}{lccc}\text { Two Year Old Trees } & \text { Each } & 10 & 100 \\ 5 \text { to } 6 \mathrm{ft} & \$ 1 . & \$ 9.00 & \$ 80 \\ 4 \text { to } 5 \mathrm{ft} & 90 \mathrm{c} & 8.00 & 70 \\ 3 \text { to } 4 \mathrm{ft} & 80 \mathrm{c} & 7.00 & 60 \\ \text { One Year Old Trees } & & & \\ 3 \text { to } 5 \mathrm{ft} & 85 \mathrm{c} & 7.50 & 65 \\ 2 \text { to } 3 \mathrm{ft} & 75 \mathrm{c} & 6.50 & 55\end{array}$

Sweet-Bing, Black Tartarian, Gov. Wood.

Sour--Compass (Cherry Plum), Dyehouse, Early Richmond, May Duke, Montmorency Large, Montmorency Ordinary? Terry Early, Wragg.

\section{PEACH}

5 to $7 \mathrm{ft}$

4 to $5 \mathrm{ft}$

3 to $4 \mathrm{ft}$

Each $\quad 10 \quad 100$

$\$ 1 . \quad \$ 9.00 \quad \$ 20$

$90 \mathrm{c} \quad 800 \quad 70$

80 c $\quad 700 \quad 60$ (n)

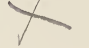

Admiral Dewey, Alton, Klack George, Bilens Late October. Uarman, Capt. Ede, Champion, Chinese Cling, Crawfor t Earlv, Crawíord Late, Crossby, Early Elberta, ${ }^{\times}$Elberta (Queen), Elberta Late, Fitzgerald, Fox's Seedling, Gold Drop, Globe, Green:boro, Heath Cling, J. H. Hale, Krummel October, Lina, Mathews Beauty, May Flower, Muir, Niagara, Red Bird Cling, R. E. Lee, Russell, Salway, Stump, Wonderful. Nectarine.

\section{PLUM}


Europeans:-Damson Free, German Prune, Green Gage, Italian Prune, Lombard, Moores Arctic, Ponds Seedling, Reine Claude, Yellow Gage.

Japanese:-Abundance, America, Burbank, Climax, Gonzales, Hale, October, Purple, Orient, Othello, Red June, Santa Rosa, Satsuma (Blood), Wickson.

Native:-Cayenne, Milton, Poul Pride, Surprise, Wild Goose.

\section{GRAPE}

Two Years Old.

All grapes, except where noted, as follows:

Each $10 \quad 100$

$25 \mathrm{c} \quad \$ 2.25 \quad \$ 20$

Black, Campbels Ey., Corıcord, Ives Seedling, Moores Early, Norton (Va. Seedling), Worden, Red, Brighton, Catawba, Delaware, Vergenese, Woodruff, Wyoming, White, Co erain, Diamond, Niagara, Martha, Pocklington. Clinton 20c, Lutie 30c, Sunrise 30c, Green Mountain 40c.

\section{CURRENTS}

$\begin{array}{lccc} & \text { Each } & 10 & 100 \\ 2 \text { Year Old (unless noted) } & 25 \mathrm{c} & \$ 2.00 & \$ 15 \\ 1 & 20 \mathrm{c} & 1.50 & 12\end{array}$

Black-Black Victoria.

Red-Cherry, Fav's Prolific, Lundon Market, Perfection 40c, Pomonia.

White-White Grape.

\section{GOOSEBERRIES}

Downing, 1 year

Each $10 \quad 100$

Houghton 1 “"

20 c $\$ 180 \quad \$ 16$

25 c $\quad 225 \quad 20$

Industry 1 “.

15 c $\quad 1.25 \quad 10$

20c $\quad 1.80 \quad 16$

35 c $\quad 300 \quad 25$

$\begin{array}{cccccc}\text { Josselyn } & 2 & \text {. . } & 40 \mathrm{c} & 350 & 30 \\ & 1 & \text {.. } & 20 \mathrm{c} & 1.80 & 16\end{array}$

$25 \mathrm{c} \quad 225 \quad 20$

$\begin{array}{llllll}\text { Pearl } & 1 & \cdots & 25 \mathrm{c} & 2.25 & 20 \\ & 2 & \cdots & 30 \mathrm{c} & 275 & 25\end{array}$

\section{BLACKBERRIES}

Early Harvest

Early King

Iceberg (White)

Mersereau

Rathburn

Snyder

Giant Himalaya

Lucretia Dewberry
Each

$12 \mathrm{c}$

$12 \mathrm{c}$

$15 \mathrm{c}$

$15 \mathrm{c}$

$12 \mathrm{c}$

$15 \mathrm{c}$

$25 \mathrm{c}$

$15 \mathrm{c}$
$10 \quad 100$

1000

$\$ 1.00 \quad \$ 5 . \quad \$ 40$

$1.00 \quad 5 . \quad 40$

$1.256 . \quad 50$

$1.256 . \quad 50$

$1.00 \quad 5 . \quad 40$

$1.256 . \quad 50$

$2.00 \quad 10 . \quad 80$

$1.00 \quad 5 . \quad 40$ 


\section{RASPBERRIES}

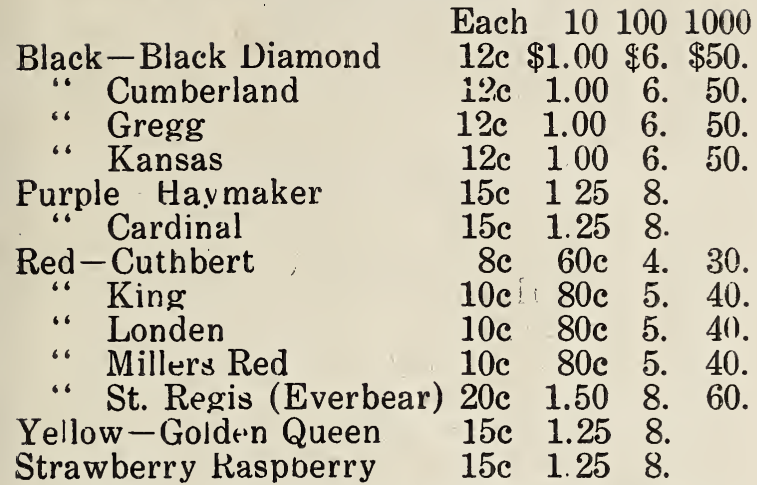

SPECIAL: - When buying blackberries, Raspberries, dewberries, strawberries, etc., we selı 25 of a kind at Hundred Rites. In digging these plants we tie 25 in a bunen.

\section{STRAWBERRIES}

Leading Varieties

$10 \quad 1001000$ Aroma (late) Climax, Corsican (medium) Excelsior (early), Gandy (late), Gl+nmary (late), Lady Tn'mpson (early), Mitchell's Early, Sen Dunlap (late), Sample (late). Wm. Kelt (medium)

Everbearıng-Superb, 10 for $\$ 1 ; 100$ for $\$ 5$.

\section{MISCELLANEOUS}

\begin{tabular}{|c|c|c|}
\hline & Each & \\
\hline Russian Mulberry (4 to $6 \mathrm{ft}$ ) & & \\
\hline Dwarf Juneberry & $40 \mathrm{c}$ & 35 \\
\hline $\begin{array}{l}\text { Highbush Cranb+rry } \\
\text { Eleagnus Longutes }\end{array}$ & $50 \mathrm{c}$ & 4.50 \\
\hline $\begin{array}{l}\text { Eleagnus Longu,es } \\
\text { Augustifolia }\end{array}$ & & 4.50 \\
\hline $\begin{array}{l}\text { Augustifolia } \\
\text { Holts Marnmuth S^ge }\end{array}$ & $50 \mathrm{c}$ & 4.50 \\
\hline $\begin{array}{l}\text { sparagus-Cunover's Colossal } \\
\text { heubarb }\end{array}$ & & $\begin{array}{r}.50 \\
1.00\end{array}$ \\
\hline
\end{tabular}

\section{ROSES}

Ramblers or Vining Roses-These are all hardy and trong tu o year old bushes. Price 50 . each except where oterl.

Ramblers-Crimson, Pink, Yellow, Whlte, Blue 75c, Flower of Fairfield or Everblooming Crim-on $R_{\uparrow}$ mbler $\$ 1$, Durothy Perkins (piuk), Write Dorotha, R+d Dorotha, Hiawatha $(\mathrm{R} \oplus d)$, ( rold Finch

Vining Ruses-Thnusarid Beauty 60c, Baltimo e Belle 40c. Prairie Queen (Red) 40c, Anna Moriah (pink) 40c.

Everblonming Roses Climbing Baby Rambler 60c: Climbing Clothilda Souport 60c, Gen. Jaqu mont (Red) 75c, Fran Karl Druschki 75c, rinihilda Soupurt 60c.

B $\leadsto$ bv Ramb! $r$-Crimson, Pink, White.

Misc llaneous - Pink Moss, Harrison's Yellow arid Sweet Briar. 


\section{Shade and Ornamental Trees}

(4 to $6 \mathrm{ft}$ except where noted)

Ailanthus (Tree or Heaven)

Each 10

Ash-European Mountain

Bırd Cherry

Birch-European White

"American Sweet

Catalpa-Speciosa

Crab-Bachlets Fiowering (3 to $5 \mathrm{ft}$ )

Cornus-Florida Dogwood

“ Blue Dogwood

“ Cornelia Cherry

“ Enylish Dogwood

Horse Chestnut-Write Fb.

$60 \mathrm{c} \$ 5$.

60 c 5 .

60 c 5 .

60 c 5 .

$50 \mathrm{c} 4$

50 c 4 .

60 c 5 .

60 e 5 .

60 c 5 .

$60 \mathrm{c} 5$.

60 c 5 .

$70 \mathrm{c} 6$.

Koelrenteria Paniculata (varnish tree) $60 \mathrm{c} 5$.

European Larch

Liquid Amber (Sweet Gum)

Locust - Black or Yellow

"Honev

Linden-American

Maple-Ash Leaved

"Norway

“ Silver

“ Scarler or Red

“ Sugar or Roch

Persimon-American

Poplars--Bolleana (silver leaved)

"Corolina

Ptelea Trifoliata (Hop Tree)

Salisburia (Maıden Hair Tree)

Tulip Tree (Yellow Poplar)

Willow-Gold Bark

“Golden H'e ping

“ Babylonica Weeping

$\$ 18$.

50c 4.

$50 \mathrm{c} 4$.

$60 \mathrm{c} 5$.

60 c 5 .

50 c 4.

70 c 6.

$50 \mathrm{c} 4$.

$50 \mathrm{c} 4$.

$50 \mathrm{c} 4$.

60 c 5.

80 c 7.

$50 \mathrm{c} 4$.

60 c 5 .

$\$ 18$.

$50 \mathrm{c} 4$.

60 c 5 .

60 c 5 .

$80 \mathrm{c} 7$.

\section{SHRUBBERY}

Each 10

Althea-Common Single

"Ardens, double, blue

“ Boule de Fen, double, red

“ Carnea Plena, double rose

“ Coelestis, single, blue

$25 \mathrm{c} \$ 2$.

$50 \mathrm{c} 4$.

$50 \mathrm{c} 4$.

$40 \mathrm{c} 3$.

$40 \mathrm{c} 3$.

“ Folins Variegata, variegated leav 80? 7.

“ Jean d'Arc, double, pure white 60c 5.

“ Lady Stanley, doutle, pink 40c 3.

“Totus Albus, single, pure white $50 \mathrm{c} 4$.

Button Bush, dwarf $50 \mathrm{c} 4$.

Calycanthus, sweet scented shrub 50c 4.

Clethra Alnifolia, swert pepper bush 50r 4 .

Cydonia Japonica, red lap. Quince 40c 3.

Dentzia-Pride of Rochester $50 \mathrm{c} 4$.

Enonymus or Strawberry Tree 50c 4.

"Jurngaens, Burning Bush 50c 4 .

Forsychea Fortunei, (rolden Bell 50c 4.

“Suspensi $40 \mathrm{c} 3$.

“ Viridissima 40c 3 .

Hydrangea - Aborsceus, Hills of Snow 50c 4 . " Paniculta Grandifolia 60c 5

Kerria Japonica or Globe Flower 40c 3.

" Variegata, variegated lea 40c 3.

“ White 
Lonicera Tartaric Alba, H. Suckle, W. 40c 3. “ "Grandiflora Rosea, P. 40c 3. " Red $40 \mathrm{c} 3$.

Philadelphus-Aurens, Golden Mock

“ Dwarf Mock Orange 40c

“ Coronarius, sweet flowered $50 \mathrm{c} 4$

“ “ Lomarius, sweet flowered Lomoin es erectus

$40 \mathrm{c} 3$.

Rhus Cotinus, Purple Fringe $\quad 50 \mathrm{c} 4$.

Spiraea-Anthony Waterer, dwarf P. 60c 5.

“ Billardi, pink 40c 3.

“ Golden Leaved 50c 4.

“ Bridle Wreath 40c 3.

“ Reevesiana, double flowered, W. 40c 3.

“Van Houttie, white 40c 3.

Syringa or Lilac-Red Persian $40 \mathrm{c} 3$. $\because$ Vulgaris, purple $\quad 40 \mathrm{c} 3$.

“ " " Alba, white 50c 3.

Viburnum Opulus, Snowball $50 \mathrm{c} 4$. - Oxvcoccus, Bush Cranberry 50c 4.

Wegelia-Candida, white 50c 4.

" Rosea, pink 40c 3.

“ Eva Rathke, red 60c 5.

“ Variegated Leaved $60 \mathrm{c} 5$.

Prunus-Pissardi, Purple Leaved Plum 80c 7. "Othello, " " " 8uc 7.

“ Trilobia, double flowering plum 80c 7.

"Amygdalus, dwarf " almon 50c 4.

Crab-Bachtel's, doubie flowering $60 \mathrm{c} 5$.

Barberry-- Japanese 40c 3. $\begin{array}{ll}50 \mathrm{c} & 4 . \\ 80 \mathrm{c} & 7 .\end{array}$

\section{VINES AND CREEPERS}

Each 10 Ampelopsis Quinqurfolia. Va. Creeper 40c $\$ 3$. Bignonia Radicans. Trumpet Vine $40 \mathrm{c} 3$. Lycium Barb rum, Matrimony Vine 40c 3. Lonicerin, Halls Honeysuckle 40c 3. “ Monthly Fragrant 40c 3. “ Scarlet Trumpet $40 \mathrm{c} 3$. Wisteria, Chinese Purple $40 \mathrm{c} 3$. Cinnamon Vine, tubers $30 \mathrm{c} 2.50$

\section{EVERGREENS}

( 12 to 18 in. -18 to 24 in -2 to $3 \mathrm{ft}-3$ to $4 \mathrm{ft}$ )

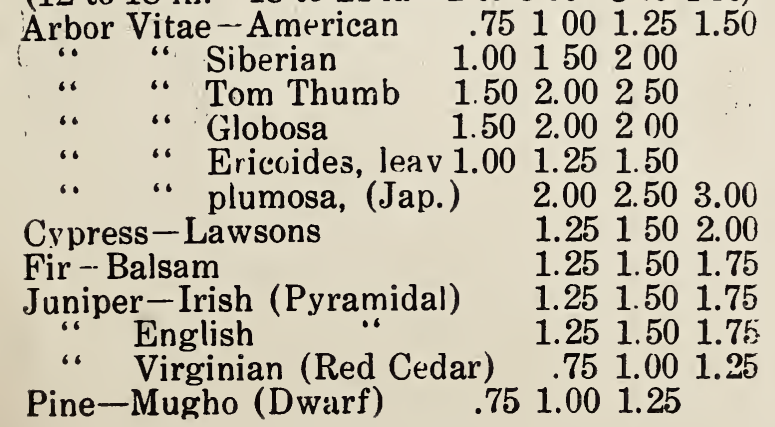


Spruce - Alba (White)

1001.251 .50

" Black

“ Colurado Blue

1.00125150

“ Black Hills

2.002 .503 .00

“ Hemlock

“ Norway

1.502 .00250

$1.001 .25 \quad 1.50$

1001.251 .50

1.001 .25150

Holly-American

Our evergreens are all well grown and beautiful specimen trees, and taken up with large ball of earth attached, that insures safe delivery.

\section{BULBS}

Cannas - Assorted Color's

Each 10

$30 \mathrm{c} \$ 250$

Dahlias - Double, rəd, pink, W. \& Y. 25c 200

“ Single. assorted

“ Pompon, purple, Red, pink

Gladioli, assorted colors

$15 \mathrm{c} 1.00$

25 c 2.50

$10 \mathrm{c} .60$

$15 \mathrm{c} 100$

Golden Glow

$\begin{array}{lll}\text { Hibiscus. assorted; red, pink, white } 25: 2.00 \\ \text { Hollyhocks, Si.gle, assorted colırs } 10 \mathrm{c} & .80\end{array}$

Hollyhocks, Sir.gle, assorted colırs $10 \mathrm{c} .80$

Hyacinthus Candicans

Iris-Japanese, purple and gold

“ English, assorted colors

20 c 150

$25 \mathrm{c} 2.00$

$20 \mathrm{c} 1.50$

“S Spanısn, “"

“German, blue and white 10c 1.50

Narcissus or Daffodils, single, double 15c 1.00

Paeonia, red, pink, white, yellow 30c 2.50

Tube Rose Excelsior Pearl $10 \mathrm{c} \quad .80$

Tulips, double, single, and Darwin

\section{HARDY PERENNIALS}

(First six named, in assorted colors) Antirhimun or Snap Dragon, Aster-Perennial

$15 \mathrm{c} 1.25$

Canterbury Bell

$15 \mathrm{c} 1.25$

15 c 1.25

Carnation Pinks

15 c 125

Columbine (Aquilegia)

20 c 150

Chrysanthemums, lirge flowers $20 \mathrm{c} 1.50$

- Pompon, small flowers 20c 150

Calladium, (Elephant Ears) Ig. bulbs 30c 250 Calla-Elliotiana, spotted leaf

Lillies, assorted

25 e 2.00

$20 \mathrm{e} 1.50$

\section{ORNAMENTAL HEDGE}

1 to $1 \frac{1}{2} \mathrm{ft}-1 \frac{1}{2}$ to $2 \mathrm{ft}-2$ to $3 \mathrm{ft}$ per $1(10$ per 100 per 100

California Privet $\$ 8 . \quad \$ 10 . \quad \$ 12$. Spirea Van Houttie $12 . \quad 15 . \quad 20$. 


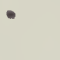




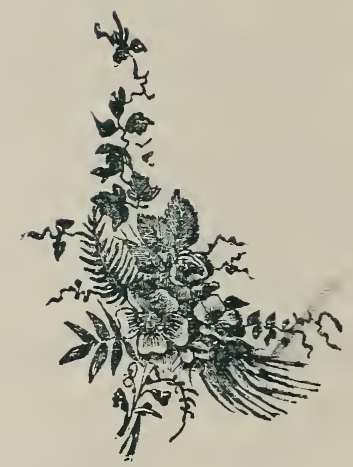

